\title{
Measuring healthcare service quality in a private hospital in a developing country by tools of Victorian patient satisfaction monitor
}

\author{
Si Dung Chu*1,2, Tan Sin Khong ${ }^{2,3}$ \\ ${ }^{1}$ Vietnam National University, Hanoi, Vietnam \\ ${ }^{2}$ European University, Switzerland \\ ${ }^{3}$ MultiMedia University, Malaysia
}

Received: January 19, 2018

Accepted: May 16, 2018

Online Published: June 20, 2018

DOI: $10.5430 /$ jha.v7n5p1

URL: https://doi.org/10.5430/jha.v7n5p1

\begin{abstract}
Objective: A survey was conducted for patient satisfaction (PS) in a private hospital in Hanoi, Vietnam using the Tools of Victorian Patient Satisfaction Monitor (VPSM) 2012.

Methods: The study obtained feedbacks from patients from December 2013 to Jan 2014. After patients finished their outpatient care at the hospital, they were asked 25 questions following the VPSM. Each question can score from 1-5.

Results: In total, 420 patients were interviewed and 396 patients completed the survey. Levels of customer satisfaction about service quality (SQ) were influenced by the VPSM with 6 items (25 score) and Cronbach alpha coefficient's 0.938 , including: (1) Access and Admission, (2) General Patient Information, (3) Treatment and Related Information, (4) Complaints Management, (5) Physical Environment, and (6) Discharge And Follow, with highly Corrected Item-Total Correlation of six constructs of PS (from 0.749 to 0.885 ). Overall, the level of highly PS in the hospital was explained almost $77.347 \%$ what could be achieved. There is no difference in the assessment of the SQ for medical examination under the "Gender" and the "level of education", but there is a difference in the assessment of the SQ between the "monthly income level" with the model of research.

Conclusions: Adjusted research model for the hospital has six constructs of levels of customer satisfaction about SQ is influenced by the VPSM with 6 items ( 25 score). The VPSM provides feedback on the SQ for medical examination of a private hospital experience from the adult outpatient's perspective at the developing nation as Vietnam country.
\end{abstract}

Key Words: Service quality, Medical examination, Patient satisfaction, Victorian Patient Satisfaction Monitor

\section{INTRODUCTION}

\subsection{Introduction of the modeled hospital}

Tri Duc General Hospital is one of the most well-known private hospitals in Vietnam. Although Vietnam is now a developing country, Tri Duc Hospital has combined advanced technology and expertise in medical service. Founded in 2007, the hospital has provided healthcare service to more than 257,000 patients, including 17,000 patients undergoing surgical procedures. Currently, Tri Duc hospital has 2 connected buildings: a 9-story for outpatient service and a 5-story for impatient with 50 beds. That hospital also developed completely with 76 doctors (including 5 medical professors) and 154 nurses. The staff's philosophy is to provide best medical service to customers. Furthermore, patients choose Tri Duc not only because of its high service quality

*Correspondence: Si Dung Chu; Email: chudungsi@gmail.com; Address: Vietnam National University, Hanoi, Vietnam. 
(SQ) but also because Tri Duc is located in a favorable site, 2.2 Selection of respondents

linked to main roads and transportation system such as buses, Patients in outpatient clinic at Tri Duc General Hospital were railways and others. chosen for the survey.

\subsection{Introduction of the VPSM}

Victorian Patient Satisfaction Monitor (VPSM) is a study that asks patients to tell how they felt about their stay in the hospital. ${ }^{[1,2]}$ The continuous monitor of patient satisfaction (PS) has been required by Victorian Government as a commitment to improve the public healthcare system. ${ }^{[3,4]}$ The results are crucial for quality managers to direct the improvement and change in healthcare service.

As VPSM proved great impact on the improvement of healthcare system, we applied that model to measure PS in Tri Duc hospital - a private hospital in Hanoi, Vietnam. After analysis, we proposed some suggestions to improve the medical SQ.

\section{Methods}

\subsection{Study area}

Patients who were examined in General clinic department at Tri Duc General Hospital.

\subsection{Tools of VPSM}

Measuring PS by Tools of VPSM (2012) contains six dimensions with 25 items: Each item can be scored from 1 to 5 . The six aspects are: Access and Admission with 5 items, General Patient information with 4 items, Treatment and Related information with 6 items, Complaints Management with 3 items, Physical Environment with 5 items, Discharge and Follow-up with 3 items (see Figure 1). ${ }^{[3,4]}$

The scores of 25 surveyed items are used to calculate the Overall Care Index (OCI). The OCI acts as the global indicator for the patients' hospital experience.

\subsubsection{Interval measurement for $S Q$ and $P S$}

This measurement has the power to measure the distance between any two points on the scale. Respondents are to provide answers on their expectations and perceptions based on the 5-point Likert scale. 1 implies SD - Strongly Disagree, 2 implies D - Disagree, 3 implies N - Neither disagree nor agree, 4 implies A - Agree, 5 implies SA - Strongly agree. ${ }^{[5]}$

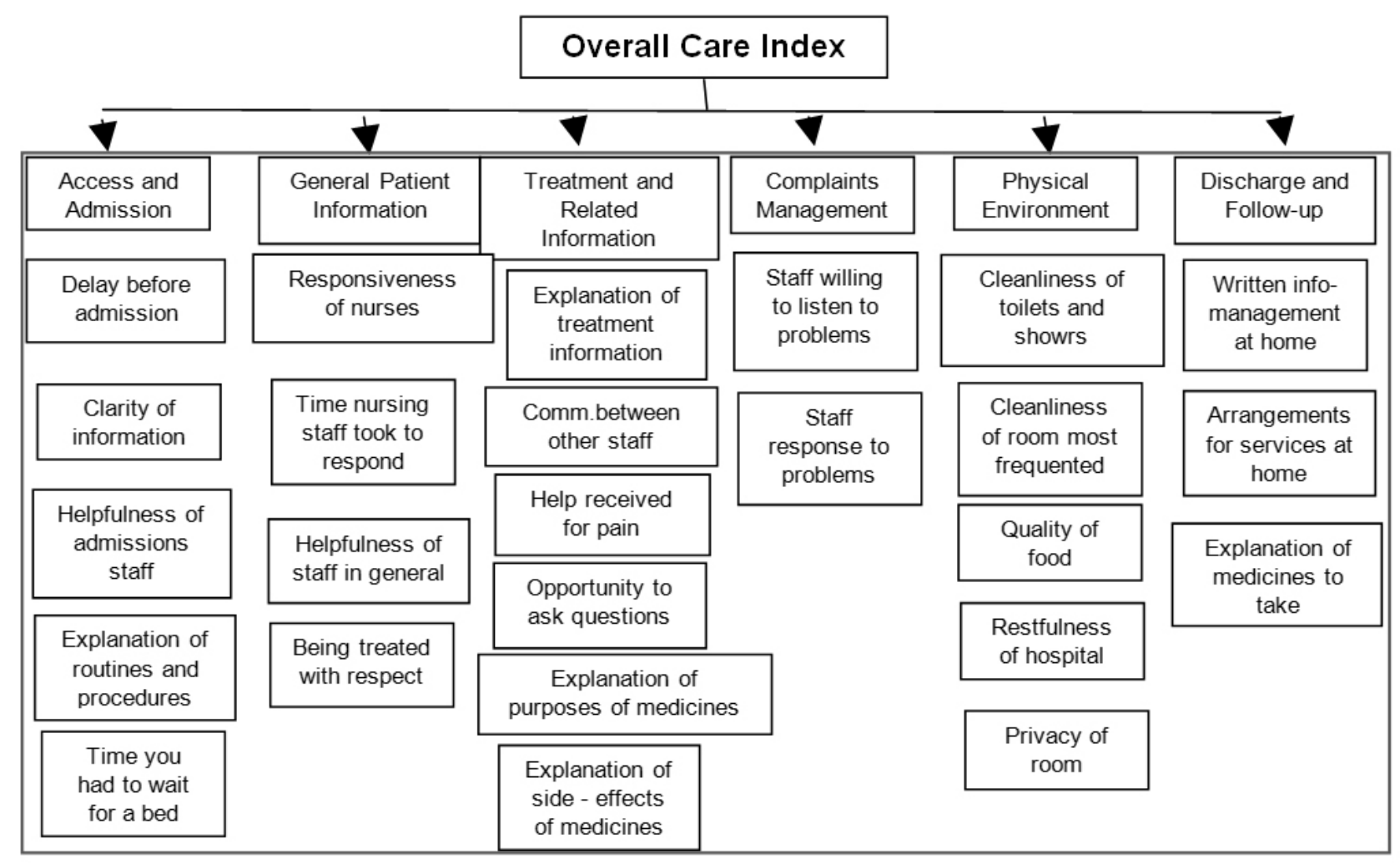

Figure 1. VPSM indices and items (Source: VPSM Annual Report 2012-12) ${ }^{[3,4]}$ 
(1) Independent variables (IV) and dependent variables (DV):

- PS in SQ is a DV.

- 6 dimensions of PS are IV of PS (DV). Measuring PS by 6 dimensions are: the 1st variable is Access and Admission (DVa: 5 variables), the 2nd variable is General Patient information (DVb: 4 variables), the 3rd variable is Treatment and Related information (DVc: 6 variables), the 4th variable is Complaints Management (DVd: 2 variables), the 5th variable is Physical Environment (DVe: 5 variables), and the 6th variable is Discharge and Follow-up (DVf: 3 variables).

\section{Demographic variables (DM):}

- DM1: Gender $(0=$ male, $1=$ female $)$

- DM2: Level of Education $(0=$ undergraduate, $1=$ Degree, $2=$ master, $3=$ Doctorate)

- DM3: Income Level: Monthly income $(0=$ under 200 USD, 1 = 201-500 USD, 2 = 501-1,000 USD, $3=1,001-2,000$ USD , 4 = above 2,000 USD)

\section{(2) Research hypotheses}

For the purpose of this research, we argue the VPSM indexes are reliable and all six dimensions of PS in SQ by the VPSM instrument are significant in the setting of health care.

\section{Demographic variables (DM):}

- H1a (Hypothesis 1a): There is a relationship between "Access and Admission" and "Service Quality".

- H1b (Hypothesis 1b): There is a relationship between "General Patient information" and "Service Quality".

- H1c (Hypothesis 1c): There is a relationship between "Treatment and Related information" and "Service Quality”.

- H1d (Hypothesis 1d): There is a relationship between "Complaints Management" and "Service Quality".

- H1e (Hypothesis 1e): There is a relationship between "Physical Environment" and "Service Quality".

- H1f (Hypothesis 1f): There is a relationship between "Discharge and Follow-up" and "Service quality".
From the analysis, some suggestions were proposed to improve the quality of healthcare, ensure PS for general clinic department at this hospital.

\section{(3) Analysis of variance (ANOVA):}

One-way ANOVA was performed to find a difference in the quality assessment of healthcare services according to demographic variables (gender, level of education, income level), with these assumptions:

\section{Demographic variables (DM):}

- (H3a): There is a difference in satisfaction levels between the genders.

- (H3b): There is a difference in satisfaction levels between the levels of education.

- $(\mathrm{H} 3 \mathrm{c})$ : There is a difference in satisfaction levels between the monthly income levels.

\section{(4) Questionnaire administration:}

Questionnaires were completed by outpatients at the modeled hospital $(n=396)$ over a period of one month.

All data analysis has been carried out with the Statistical Package for Social Sciences (IBM SPSS 21.0). ${ }^{[6,7]}$

\section{RESUlts}

From the samples characteristics in the private hospital: 420 questionnaires were distributed, the rate of completion is $94.29 \%(n=396)$. There is a 396 questionnaire are completed, frequency distribution of gender in the hospital are 173 male $(43.7 \%)$ and 223 female (56.3\%).

\subsection{Reliability (Cornbach alpha) and average of PS vari- ables}

Patients were basically satisfy the needs of the patient but still not really satisfied. The satisfaction of patients using health care services in private hospitals is to achieve high satisfaction.

Table 1. Descriptive statistics of the PS constructs in the private hospital

\begin{tabular}{llll}
\hline Dimensions & N & Mean $\pm \boldsymbol{S D}$ & Reliability (Cornbach Alpha) \\
\hline Access and Admission (DVa) & 396 & $3.9965 \pm 0.58043$ & 0.883 \\
General Patient information (DVb) & 396 & $4.1995 \pm 0.60219$ & 0.895 \\
Treatment and Related information (DVc) & 396 & $4.0299 \pm 0.59014$ & 0.896 \\
Complaints Management (DVd) & 396 & $4.1679 \pm 0.63162$ & 0.900 \\
Physical Environment (DVe) & 396 & $3.8212 \pm 0.73382$ & 0.912 \\
Discharge and Follow-up (DVf) & 396 & $4.0118 \pm 0.64903$ & 0.896 \\
\hline
\end{tabular}


Basically, patients satisfied to some extent. Variables Dva, DVb, DVc, DVd, Dve, and DVf were variable are the used in this study to measure patient satisfaction. These many items as in Figure 1 were measured againsts five point Likert scale ranging from $1=$ strongly disagree to $5=$ strongly agree. The reliability coefficient, Cronbach Alpha for these construct of the private hospital are $0.883,0.895,0.896,0.900,0.912$, and 0.896 respectively (see Table 1 ).

\subsection{Exploratory factor analysis (EFA) for private hospi- tal}

After performing EFA of 6 components ( 25 scores), we have one component extracted with six factors (25 scores) are drawn (DVa, DVb, DVc, DVd, DVe, DVf) (see Table 2 and Figure 2).

\subsection{Cronbach alpha of factor and model for private hos- pital}

The reliability coefficient for PS was calculated based on the Cronbach alpha coefficient for the six variables of PS, as can be seen in Table 3: Cronbach alpha coefficient $=0.938$ and all the variable in PS have coefficients of Corrected item Total Correlation are greater than 0.3 (Coefficients Corrected Item-Total Correlation of six constructs of PS are DVa = $0.830 ; \mathrm{DVb}=0.811 ; \mathrm{DVc}=0.885 ; \mathrm{DVd}=0.815 ; \mathrm{DVe}=$ 0.749 and DVf $=0.838$ ), satisfactory inspection, thereby ensuring conditions for inclusion in the next model analysis.

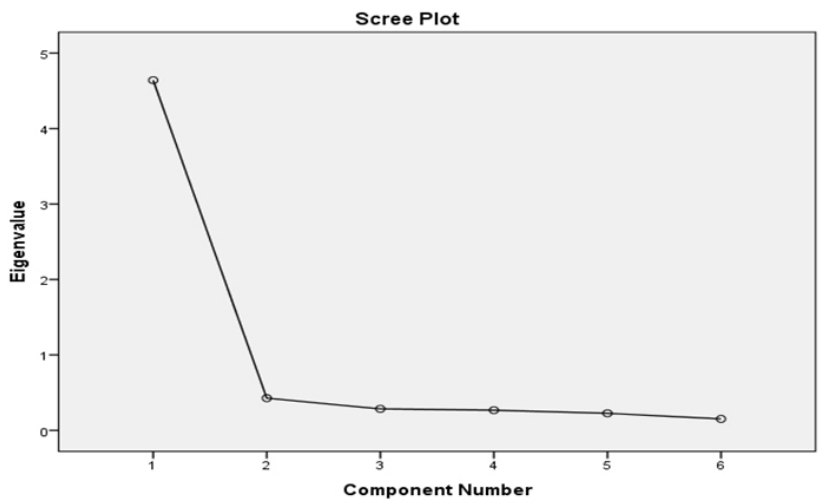

Figure 2. Component number

Table 2. EFA of PS of the results in the private hospital

\begin{tabular}{|c|c|c|c|c|c|c|}
\hline \multirow{2}{*}{ Component } & \multicolumn{3}{|c|}{ Initial Eigenvalues } & \multicolumn{3}{|c|}{ Extraction Sums of Squared Loadings } \\
\hline & Total & \% of Variance & Cumulative \% & Total & \% of Variance & Cumulative \% \\
\hline 1 & 4.641 & 77.347 & 77.347 & 4.641 & 77.347 & 77.347 \\
\hline 2 & 0.427 & 7.118 & 84.464 & & & \\
\hline 3 & 0.285 & 4.756 & 89.220 & & & \\
\hline 4 & 0.268 & 4.466 & 93.686 & & & \\
\hline 5 & 0.227 & 3.777 & 97.463 & & & \\
\hline 6 & 0.152 & 2.537 & 100.000 & & & \\
\hline \multicolumn{7}{|c|}{ Extraction Method: Principal Component Analysis } \\
\hline \multicolumn{7}{|c|}{ KMO and Bartlett's Test } \\
\hline \multicolumn{6}{|c|}{ Kaiser-Meyer-Olkin Measure of Sampling Adequacy } & 0.917 \\
\hline \multirow{3}{*}{\multicolumn{3}{|c|}{ Bartlett’s Test of Sphericity }} & Approx. Chi-Square & & & $2,060.367$ \\
\hline & & & Df & & & 15 \\
\hline & & & Sig. & & & 0.000 \\
\hline \multicolumn{7}{|c|}{ Component Matrix $^{*}$} \\
\hline & & & \multirow{2}{*}{\multicolumn{4}{|c|}{$\begin{array}{l}\text { Component } \\
1\end{array}$}} \\
\hline & & & & & & \\
\hline \multicolumn{3}{|l|}{ DVa } & \multicolumn{4}{|l|}{0.885} \\
\hline \multicolumn{3}{|l|}{$\mathrm{DVb}$} & \multicolumn{4}{|l|}{0.875} \\
\hline \multicolumn{3}{|l|}{ DVc } & \multicolumn{4}{|l|}{0.925} \\
\hline \multicolumn{3}{|l|}{ DVe } & \multicolumn{4}{|l|}{0.822} \\
\hline \multicolumn{3}{|l|}{ DVf } & \multicolumn{4}{|l|}{0.891} \\
\hline \multicolumn{3}{|l|}{ DVd } & 0.875 & & & \\
\hline \multicolumn{7}{|c|}{$\begin{array}{l}\text { Extraction Method: Principal Component Analysis. } \\
* .1 \text { components extracted. }\end{array}$} \\
\hline \multicolumn{7}{|c|}{ Rotated Component Matrix ${ }^{\#}$} \\
\hline
\end{tabular}


Table 3. Cronbach alpha of PS of the results in the private hospital

\begin{tabular}{lllll}
\hline Items & $\begin{array}{l}\text { Scale Mean if Item } \\
\text { Deleted }\end{array}$ & $\begin{array}{l}\text { Scale Variance if Item } \\
\text { Deleted }\end{array}$ & $\begin{array}{l}\text { Corrected Item-Total } \\
\text { Correlation }\end{array}$ & $\begin{array}{l}\text { Cronbach's Alpha if Item } \\
\text { Deleted }\end{array}$ \\
\hline $\mathrm{DVa}$ & 20.2303 & 7.980 & 0.830 & 0.926 \\
$\mathrm{DVb}$ & 20.0273 & 7.926 & 0.811 & 0.928 \\
$\mathrm{DVc}$ & 20.1969 & 7.778 & 0.885 & 0.919 \\
$\mathrm{DVd}$ & 20.0588 & 7.769 & 0.815 & 0.927 \\
$\mathrm{DVe}$ & 20.4056 & 7.493 & 0.749 & 0.938 \\
$\mathrm{DVf}$ & 20.2150 & 7.616 & 0.838 & 0.924 \\
\hline
\end{tabular}

Note. Reliability staitistics of PS $(n=6)$ had Cronbach's alpha: 0.938

Table 4. Summary of hypotheses findings in the private hospital

\begin{tabular}{|c|c|}
\hline Hypothesis & Result \\
\hline (H1a): There is a relationship between "Access and Admission" (DVa) and Service quality (PS). & Supported \\
\hline (H1b): There is a relationship between "General patient information and Service Quality" (DVb) and Service quality (PS). & Supported \\
\hline (H1c): There is a relationship between "Related information" (DVc) and Service quality (PS). & Supported \\
\hline (H1d): There is a relationship between "Complaints management" factor (DVd) and Service quality (PS). & Supported \\
\hline (H1e): There is a relationship between "Physical Environment" (DVe) and Patient satisfaction (PS). & Supported \\
\hline (H1f): There is a relationship between "Discharge and Follow-up" (DVf) and Patient satisfaction (PS). & Supported \\
\hline
\end{tabular}

\subsection{Adjusted research model and hypothesis for private hospital}

Through the above analysis results showed that 6 factors (components) of the original scale service quality after performing factor analysis, worth six factors distinguish drawn, which were six components as above (see Table 3): DVa, DVb, DVc, DVd, DVe, DVf components. That ensures conditions for adjusted research model and hypothesis.

Thus, the initial research model through factor analysis results are adjusted in Table 4.

\subsection{Analysis of variance ANOVA for private hospital}

The result of the analysis of variance ANOVA gender indicated that $F$ value had no significant (Sig. $=0.115$, greater than 0.05). The result of the analysis of variance ANOVA education groups showed that $\mathrm{F}$ value have no significant (Sig. $=0.364$, greater than 0.05 ), therefore it had no significant difference in patient satisfaction between different genders as well as between the education groups, with confidence Interval Level in 95\% (0.05). Thus, (H3a) hypothesis and (H3b) hypothesis are rejected.

The result of the analysis of variance ANOVA income level groups demonstrated that $F$ value had significant (Sig. = 0.000 , less than 0.05). That can be concluded that there was significant difference in patient satisfaction between different income-level groups, with confidence Interval Level in 95\% (0.05). Thus, (H3c) hypothesis is supported.

\section{Discussion}

\subsection{Reliability (Cronbach alpha) of variable}

Reliability of the instrument helped to provide consistency in the results and the Cronbach alpha was used to measure the reliability of the data. Overall Cronbach Alpha of private hospital data along with service quality construct provided values greater than 0.60 (see Table 1), which is acceptable. ${ }^{[7,8]}$

\subsection{Exploratory factor analysis (EFA)}

Factor analysis discovered EFA with PS variable group:

Continuing to performing PS (dependent variable are) analysis of 6 factors as above (DVa, DVb, DVc, DVd, DVe, DVf), we have 1 element drawn and obtain results (see Table 2): $\mathrm{KMO}=0.917(>0.5)$, sig. $=0.000(<0.05)$ in Bartlett's test of sphericity. Therefore, it is suitable for the conditions of factor analysis. One factor (Only one component was extracted) is drawn with variance extracted at $77.347 \%$ (see Table 2). ${ }^{[6,7]}$

The model explains almost $77.347 \%$ only of the variance. The rest $(22.653 \%)$ could not be explained by the variables included in the analysis. As can be seen in Table 2 (Total Variance Explained), the rotation converged in 6 iterations that were consistent with the framework the researchers had formulated in the current research. Therefore, this model was proven to be the most appropriate measurement of functional quality for the current field of research. Thus, factor analysis has demonstrated that the model is constructed from 
6 major constructs defined in Table 2 and Figure 2 (Demonstrating Rotated Component Matrix and Constructs of the Research). ${ }^{[6,7]}$

As can be seen in Table 2, The rotation converged in 6 iterations that were consistent with the framework the researchers had formulated in the current research. Thus, this model was proven to be the most appropriate measurement for PS for the current field of research (see Table 2).

\subsection{Cronbach alpha of factors and adjusted research model for hospital}

The reliability coefficient, PS is brought to checks in the Cronbach alpha coefficient for the six constructs of PS, test results (see Table 3) were showed that Cronbach alpha coefficient $=0.983$ and all the variable in PS have coefficients of Corrected item - Total Correlation is greater than 0.3 (Coefficients Corrected Item-Total Correlation of six construct of PS are DVa $=0.830, \mathrm{DVb}=0.811, \mathrm{DVc}=0.885, \mathrm{DVd}$ $=0.815, \mathrm{DVe}=0.749, \mathrm{DVf}=0.838)$. Thus, that ensures conditions for inclusion in the next model analysis. ${ }^{[6,7]}$

\subsection{Adjusted research model and hypothesis for private hospital}

Through the above analysis results showed that 6 factors (components) of the original scale SQ after performing factor analysis, worth six factors distinguish drawn, which were the initial research model through factor analysis results are adjusted as follows (see Table 3 ).

In the hospital, this is the most powerful factor which strongly influences the quality of healthcare service and in turn customer satisfaction. This is also the core element of satisfaction with medical care, a hospital should be promoted, and especially doctors need qualified professionals, better skills and continuously improve in qualifications. ${ }^{[8]}$ The staff of the hospital departments should also enhance professional and service attitude better ensure service capacity associated with the customer's interests. There are also very important factors that hospital management needs to attend in the construction of the development strategy; therefore, the management of hospital need to focus more on investing in upgrading establishment and medical equipment, upgrading working environment and admission system as well as expanding the size of the hospital, especially, the medical examination area. That will ensure good environment in hospitals. The hospital administration needs to organize some counselling to patients before they arrive at the hospital and follow the clients' progress when they are at home.

In fact, Australian is a developed country, the use of the VPSM with 6 items (25 scores) are common and many developed countries have used to the tool. That evidence con6 firmed that the VPSM is a strong tool in the measurement of service quality by levels of customer satisfaction. ${ }^{[2-4]}$ Our research utilized the tool of VPSM in developing countries as Vietnam country, showing that the VPSM can become a strong tool when applying for the developing countries; ${ }^{[1,2]}$ We can use it in measuring service quality and medical examination in most countries include developed and developing countries. $^{[2]}$

\subsection{Analysis of variance ANOVA for private hospital}

Group theory about the difference in quality evaluation of healthcare services quality according to demographic variables (gender, level of education, income level). After analysis of variance ANOVA for private hospital, result of the analysis of variance ANOVA gender, education groups, and income level groups showed that two hypothesis (H3a, H3b) are rejected, it's meaning that researchers can see the no difference in the assessing of the service quality respectively under the "Gender", between the "level of education" in the model of private hospital. Besides, the only hypothesis (H3c) is supported, we can see the difference in the assessing of the service quality respectively between the "monthly income levels" with the model of analysis. Therefore, the hospital management needs to put more attention to the cost which is a major issue that should be considered, so the service will be suitable for all customer groups. Also, the hospital needs to build a policy that can better meet the requirements of most patients during medical care as well as the admission process.

\section{Conclusions}

This patient satisfaction survey by tools of the VPSM is the firth of its kind for private hospitals in Vietnam's one developing country; Research indicated the levels of customer satisfaction about service quality which is influenced by the VPSM with 6 items (25 scores): Access and Admission, General Patient information, Treatment and Related information, Complaints Management, Physical Environment, and Discharge and Follow dimension. Therefore, we hope that the Tool of VPSM can be used for measuring the quality of medical examination as well as service quality in many countries including developed and developing countries. While the survey revealed depressing feedback, the motivation of the senior management to identify areas of concern and measure patient satisfaction is a step in the right direction. There would not be any scope to improve the services unless such bold steps at measuring client satisfaction are pursued. Perhaps, repeating such studies at regular interval of say one month or per three months will be a useful guide for material intervention in development strategy. There is no difference in the assessing of the service quality under the "Gender", 
between the "levels of education", but there is a difference in the assessing quality of the medical examination service between the "monthly income levels" with the model of this hospital.

CONFlicts OF INTEREST Disclosure

The authors declare they have no conflicts of interest.

\section{REFERENCES}

[1] Andaleeb SS. Service Quality Perceptions and Patient Satisfaction: A study of Hospitals in a Developing Country. Social Science \& Medicine. 2001; 52(9): 1359-1370. https ://doi.org/10.1016/ S0277-9536(00) 00235-5

[2] Deardorff AV. Developing Country Growth and Developed Country Response. Discussion Paper. 2000; 462.

[3] NSQHS Standards. Natioanal Safety and Quality Health Service Standards: Australian Commission on Safety and Quality in Healthcare. Endorsed by Australian Health Ministers. September 2011.

[4] Victorian Government health Information. VPSM Survey Process - Victorian Patient Satisfaction Monitor, Australia. 2013. Available from: http://www.health.vic.gov.au/consumer

[5] Shahin A. SERVQUAL and Model of Service Quality Gaps: A Framework for Determining and Prioritizing Critical Factors in Delivering Quality Services, University of Isfahan, Iran; 2010.

[6] Khelifa M. Factor Analysis. Zayed University Office of Research SPSS for Windows ${ }^{\circledR}$ 2009; 2009.

[7] Khelifa M. Multiple Linear Regression Analysis. Zayed University Office of Research SPSS for Windows ${ }^{\circledR}$ 2009; 2009.

[8] Debono D, Travaglia J. Complaints and patient satisfaction: a comprehensive review of the literature. Centre for clinical Governance Research in Health, University of New South Wales, Sydney Australia. 2009; 1-38. 